\title{
Numerical Simulation of Unsteady-State Flow in Dual Porous Coalbed Methane Horizontal Wells with Complex Boundary Conditions
}

\author{
Cheng-yong Li, ${ }^{1}$ Jun Zhou, ${ }^{1}$ Xiang-yi Yi, ${ }^{2}$ Yi Luo, ${ }^{1}$ and Ping-zhi Gong ${ }^{3}$ \\ ${ }^{1}$ Chengdu University of Technology, Chengdu, Sichuan 610059, China \\ ${ }^{2}$ State Key Laboratory of Oil and Gas Reservoir Geology and Exploitation, Chengdu, Sichuan 610059, China \\ ${ }^{3}$ CNOOC China Limited, Tianjin Branch, Tanggu 300452, China \\ Correspondence should be addressed to Jun Zhou; zhoucdut2012@126.com
}

Received 20 November 2014; Revised 26 January 2015; Accepted 27 March 2015

Academic Editor: Charles M. Drain

Copyright (c) 2015 Cheng-yong Li et al. This is an open access article distributed under the Creative Commons Attribution License, which permits unrestricted use, distribution, and reproduction in any medium, provided the original work is properly cited.

\begin{abstract}
The bottom-hole pressure response which can reflect the gas flow characteristics is important to study. A mathematical model for description of gas from porous coalbed methane (CBM) reservoirs with complex boundary conditions flowing into horizontal wells has been developed. Meanwhile, basic solution of boundary elements has been acquired by combination of Lord Kelvin point source solution, the integral of Bessel function, and Poisson superimpose formula for CBM horizontal wells with complex boundary conditions. Using this model, type curves of dimensionless pressure and pressure derivative are obtained, and flow characteristics of horizontal wells in complex boundary reservoirs and relevant factors are accordingly analyzed.
\end{abstract}

\section{Introduction}

Coalbed methane (CBM) is a kind of green and clean energy. The development and utilization of coalbed methane could not only relieve the tense situation of conventional oil and gas in supply, but also reduce the atmospheric environment pollution.

Different from conventional gas reservoirs, the migration mechanism of gas in coal is more complex and diverse [1]. Coal is dual porous media reservoir, matrix is main storage space of CBM adsorption, and fractures are main transport routes of CBM diffusion-seepage. Analyzing bottom-hole pressure helps to figure out CBM production status. Ertekin and Sung [2] established a nonequilibrium adsorption nonsteady seepage flow model in dual porosity media with Henry's law. Applying Fick's law, Anbarci and Ertekin [3] suggested a single-phase CBM seepage flow mathematical model considering pseudosteady and unsteady diffusion phenomenon. Clarkson and Bustin $[4,5]$ put forward a new double diffusion model, which assumes that adsorption occurs only in micropores and conforms to the law of nonlinear adsorption. The macropores accumulate the free gas or provide channels of gas migration between micropores and fractures. Reeve [6] proposed a new gas-water two-phase triple-porosity dual-seepage flow mathematical model, and this model can increase the third matrix pore system; however, the double-permeability model is very complex and difficult to describe and calculate. Tong et al. [7-9] introduced permeability modulus considering the deformation of coal and developed a pseudosteady diffusion nonequilibrium adsorption nonsteady seepage mathematical model. Hu et al. [10] established a gas-water two-phase percolation mathematical model of well test interpretation for CBM reservoirs, and its correctness has been verified by simulation of CBM seepage flow characteristics. Clarkson et al. [11] introduced pseudopressure function to analyze production of gas and water flow performance using the numerical simulation method. Aminian and Ameri [12] set up a function to predict gas production based on storage and transport mechanisms in CBM reservoirs. Recently, Cai and Yu [13] introduced 
the fractal theory to study the enhancing recovery mechanism in natural gas-saturated porous media by spontaneous imbibition effect.

In order to improve the production, a large number of horizontal wells have been used in the CBM reservoirs. Sung and Ertekin [14-16] established a two-dimensional, twophase, multiwell gas-water flow model, and this model has the ability to simulate multiple horizontal wells. Engler and Rajtar [17-19] established a mathematical model of single-phase gas flow in the horizontal wells, and the analytical solutions are given for the horizontal well pressure drop and pressure recovery. Wang et al. [20] established a mathematical model, in which anisotropy formation heterogeneity, permeability stress sensitivity, and influence of wellbore pressure drop on directional pinnate horizontal wells in CBM reservoirs are considered. Nie et al. [21] deduced CBM flow equations based on Langmuir adsorption in matrix and Darcy flow in fracture and analyzed the transient transport characteristics of gas from CBM reservoirs to horizontal wells.

Generally, the theories of calculating reservoir and bottom-hole pressures which can reflect the gas flow characteristics are mostly based on homogeneous reservoirs and regular geometry, such as infinite boundary or circular boundary. Outer boundary conditions of a reservoir have also been simplified; it is generally regarded as simple situations as constant pressure or closed boundary. However, influenced by characteristics of geological structures, the true reservoirs usually have complex and diversiform boundaries. In this case, the conventional flow theories and solving methods could do nothing to calculate reservoir or bottom-hole pressures with mixed boundary conditions.

The boundary element method (BEM) is a numerical computational method of solving linear partial differential equations developed after the better-known finite element method (FEM) and finite difference method (FDM). The BEM could be able to reduce dimension and save computer memory and running time. Coupling with the BEM [22], bottom-hole pressures and complicated flow characteristics under the condition of irregularly shaped area with mixed boundary could be calculated and analyzed. Numbere and Tiab [23] developed a streamline simulation with the BEM for homogeneous or partly homogeneous reservoirs with irregular boundary to make the simulation match physical model better. Kikani and Horne [24] employed the BEM to analyze the transient pressure response in reservoirs with arbitrary boundary and developed two formulas, namely, convolution formula and Laplace domain space formula, to solve transient fluid flow through porous medium in homogeneous reservoirs. Hou et al. [25] used the BEM to simulate flow line map in homogeneous reservoirs with irregular boundary. Chaiyo et al. [26] used the BEM to solve free boundary saturated seepage problem. Rafiezadeh and Ataie-Ashtiani [27] studied the flow mechanism in anisotropic media by threedimensional boundary elements.

In this paper, a mathematical model is developed to describe gas flow in horizontal wells in CBM reservoirs based on the theory of fluid flow through porous. The type curves of pressure derivative, characteristics of gas flow with complex external boundary, and relevant affecting factors are analyzed.

\section{Physical Model of Gas Flow}

Coal reservoir is a dual porous medium composed of matrix and fracture. The matrix is the main reservoir space of coalbed methane (Figure 1(a)), and the fracture is the flow channel of fluid (Figure 1(b)). The average pore size of CBM reservoirs is much smaller than those of conventional reservoirs. Pore size can be divided into three categories [28]: macropore (aperture $>20 \mathrm{~nm})$, mesopore $(2 \mathrm{~nm}<$ aperture $<$ $20 \mathrm{~nm}$ ), and micropore (aperture $<2 \mathrm{~nm}$ ). The small porosity of coal leads to the great specific surface area. Hence, methane could be strongly adsorbed, resulting in the fact that the content of CBM is far more than its pore volume.

According to the reservoir dual pore structure of coal, a physical model is set up for gas flow. The migration of gas in coal is shown in Figure 2. In the production of CBM, formation pressure keeps declining. When formation pressure drops below the critical desorption pressure, CBM starts to desorb from the coal matrix surface. Meanwhile, the original state of equilibrium is broken, causing the flow of gas in fracture. This process is very similar to spontaneous imbibition in fractured porous media [29].

To facilitate the derivation, it is assumed that the length of a horizontal well is $L$, the center location of the horizontal well is $\left(x_{w}, y_{w}, z_{w}\right)$, and the thickness of a CBM reservoir is $h$. In this paper, gas flow into horizontal wells with closed boundary (Figure 3(a)), constant pressure boundary (Figure 3(b)), and mixed boundary (Figure 3(c)) is considered.

The fundamental assumptions are as follows:

(1) CBM diffuses directly from matrix to fracture, and the process of diffusion is unsteady.

(2) Gas flow in fracture is radial laminar flow, in agreement with Darcy's law.

(3) Only single-phase gas flow exists in coal.

(4) The effects of gravity and capillary force are negligible.

(5) The effects of temperature are negligible.

(6) CBM isothermal adsorption process is in line with the Langmuir isotherm adsorption law, and the initial state conforms to the isothermal adsorption curve.

(7) Radius of the gas well is regarded infinitesimal, and gas well production is constant.

\section{Mathematical Model}

3.1. Gas Diffusion Model in Matrix. In combination with the mass conservation equation with the second Fick's diffusion law, the change of gas concentration with time is given by

$$
\phi \frac{\partial(c)}{\partial t}=\nabla\left(D^{\prime} \nabla c\right) .
$$




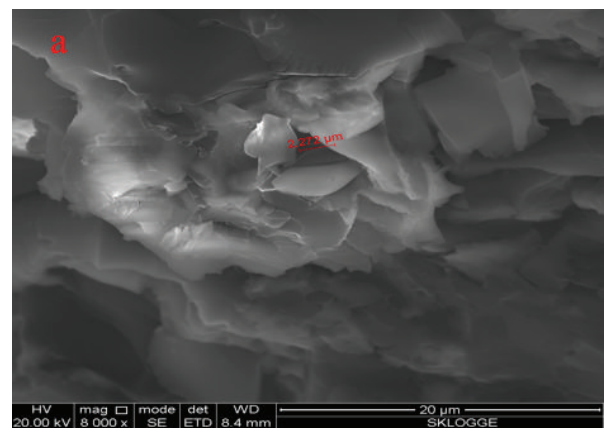

(a)

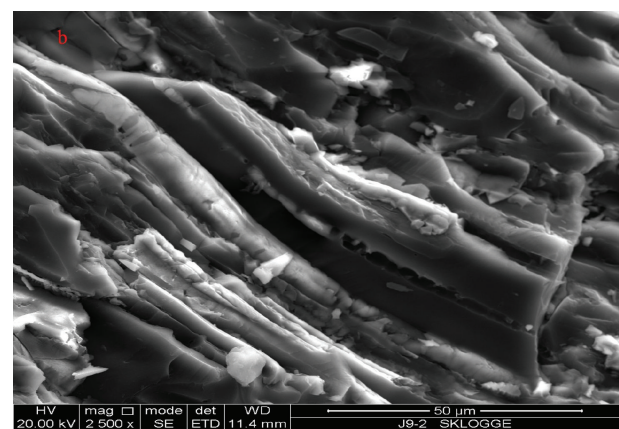

(b)

Figure 1: Pore characteristics of coal ((a) micropore; (b) fracture).
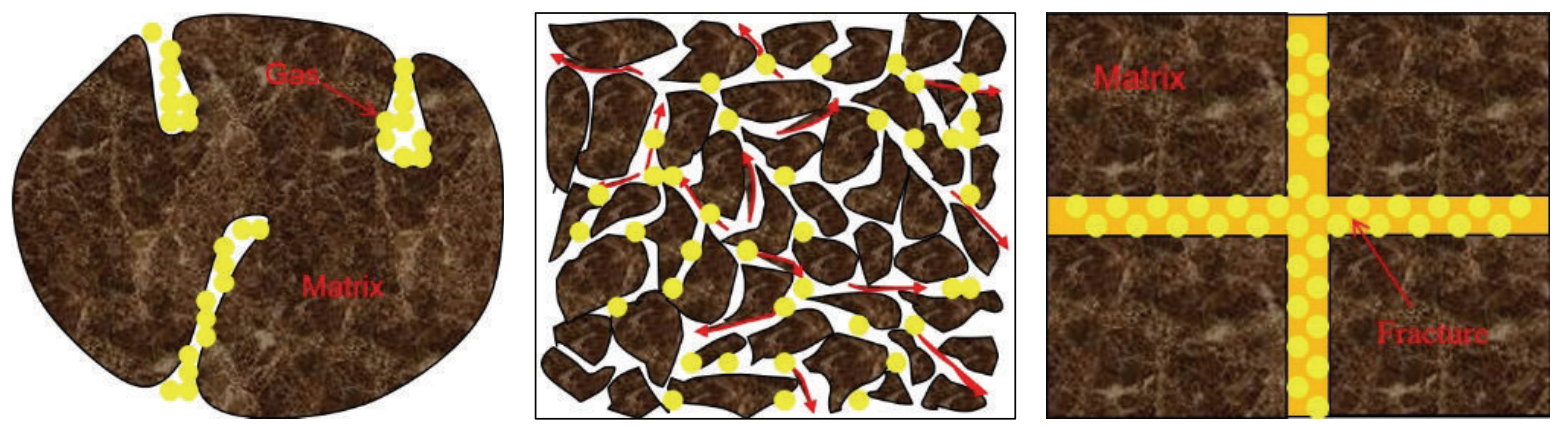

FIGURE 2: Desorption and diffusion in the CBM reservoirs.

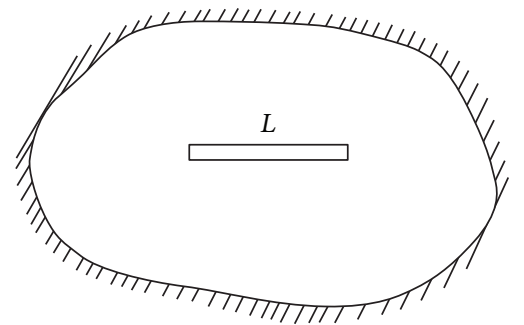

(a)

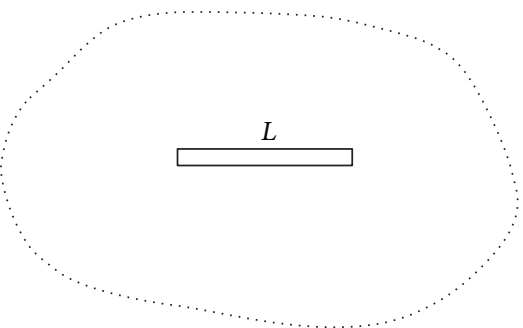

(b)

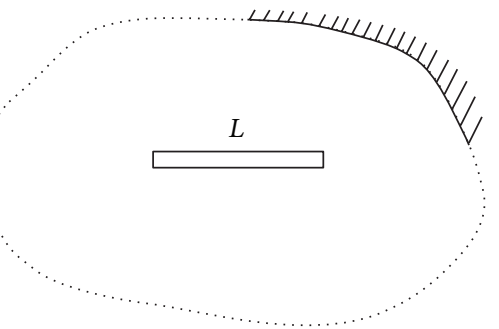

(c)

FigURE 3: Horizontal wells with different boundaries ((a) closed boundary; (b) constant pressure boundary; (c) mixed boundary).

The planar radial flow equation is as follows:

$$
\frac{\partial c}{\partial t}=\frac{D}{r_{i}^{I}} \frac{\partial}{\partial r_{i}}\left(r_{i}^{I} \frac{\partial c}{\partial r_{i}}\right)
$$

The dimensionless equation of gas diffusion in matrix is

$$
\frac{1}{r_{i D}^{2}} \frac{\partial}{\partial r_{i D}}\left(r_{i D}{ }^{2} \frac{\partial c_{D}}{\partial r_{i D}}\right)=\lambda_{1} \frac{\partial c_{D}}{\partial t_{D}},
$$

where $r_{i D}$ is the dimensionless radius defined by $r_{i D}=r_{i} / R$; $c_{D}$ is the dimensionless diffusion concentration defined by $c_{D}=c-c_{i} ; t_{D}$ is the dimensionless time defined by $t_{D}=$ $3.6 k t / \theta r_{w}{ }^{2} ; \lambda_{1}$ is interporosity flow coefficient defined by $\lambda_{1}=3.6 k R^{2} / \theta D r_{w}{ }^{2} ; \theta$ is comprehensive storage coefficient defined by $\theta=\phi_{f} c_{t} \mu+6 p_{s c} T / q_{D} T_{s c} \psi_{i} ; q_{D}$ is the dimensionless production defined by $q_{D}=1.842 \times 10^{-3} q_{s c} p_{s c} T / k h T_{s c} \psi_{i}$; $c_{i}$ is the initial concentration of gas, $\mathrm{kg} / \mathrm{m}^{3} ; k$ is the permeability, $\mu \mathrm{m}^{2}$; and $\psi_{i}$ is the pseudopressure.

3.2. Gas Flow Model in Matrix and Fracture. In the process of diffusion of CBM, the concentration often changes. Therefore, unsteady diffusion equation which accords with actual situation is used.

Free gas concentration is as follows:

$$
c_{1}=\rho_{1} \phi=\frac{M p \phi}{R T Z} .
$$

Concentration of the adsorbed gas is

$$
c_{2}=V_{L} \frac{P}{P_{L}+P} \text {. }
$$


Gas concentration in coal is

$$
c=\frac{M p \phi}{R T Z}+V_{L} \frac{P}{P_{L}+P} .
$$

Volume of gas desorption from coal is

$$
q_{d}=-\frac{\partial M_{d}}{\partial t}=-\rho_{s c} \frac{\partial V_{d}}{\partial t},
$$

where $M_{d}$ is the gas mass under standard condition, $\mathrm{kg} / \mathrm{m}^{3}$; $V_{d}$ is the volume of gas adsorption in coal under standard condition, $\mathrm{m}^{3} / \mathrm{m}^{3} \cdot \rho_{s c}$ is the density of gas under standard condition, $\mathrm{kg} / \mathrm{m}^{3}$.

Density of gas is defined as

$$
\rho_{s c}=\frac{M p_{s c}}{z R T_{s c}}
$$

Spherical matrix has the following relationship:

$$
\frac{\partial V_{d}}{\partial t}=-\frac{A}{V} J=-\frac{A}{V} D \frac{\partial c}{\partial r_{i}}=\frac{3 D}{R} \frac{\partial c}{\partial r_{i}},
$$

where $V$ is the volume of coal matrix, $\mathrm{m}^{3} ; A$ is the surface area of coal matrix, $\mathrm{m}^{2} ; D$ is the mass diffusion coefficient, $\mathrm{m}^{2} / \mathrm{s}$; $J$ is the diffusion flux, $\mathrm{g} / \mathrm{m}^{2} \mathrm{~s}$.

Combining (7), (8), and (9), the volume of gas desorption is given by

$$
q_{d}=\frac{M p_{s c}}{R T_{s c}} \frac{3 D}{R} \frac{\partial c}{\partial r_{i}}
$$

Based on the basic principle of material balance, the governing equations of gas flow in fracture system are given by

$$
\frac{1}{r} \frac{\partial}{\partial r}\left(r \rho \frac{k}{\mu_{g}} \frac{\partial p}{\partial r}\right)+q_{d}=\rho \phi C_{t} \frac{\partial p}{\partial t} .
$$

Equation (11) is simplified into the following equation:

$$
\frac{1}{r_{D}} \frac{\partial}{\partial r_{D}}\left(r_{D} \frac{\partial \psi_{D}}{\partial r_{D}}\right)+\frac{(1-\omega)}{\lambda} \frac{\partial c_{D}}{\partial r_{i D}}=\omega \frac{\partial \psi_{D}}{\partial t_{D}},
$$

where $\omega$ is the fracture storage ratio given by $\omega=\left(\phi c_{t} \mu\right) / \theta ; \lambda$ is the diffusion coefficient given by $\lambda=3.6 k \tau / \theta r_{w}{ }^{2} ; \psi_{D}$ is the dimensionless pseudopressure given by $\psi_{D}=\left(\psi_{i}-\psi\right) / \psi_{i} q_{D}$; $\tau$ is the adsorption time given by $\tau=R^{2} / D$.

3.3. Mathematical Model Solution. Diffusion and governing equations of gas flow in fracture are combined as follows:

$$
\begin{aligned}
& \frac{1}{r_{i D}^{2}} \frac{\partial}{\partial r_{i D}}\left(r_{i D}^{2} \frac{\partial c_{D}}{\partial r_{i D}}\right)=\lambda \frac{\partial c_{D}}{\partial t_{D}}, \\
& \quad \text { (diffusion in matrix), } \\
& \frac{1}{r_{D}} \frac{\partial}{\partial r_{D}}\left(r_{D} \frac{\partial \psi_{D}}{\partial r_{D}}\right)+\frac{(1-\omega)}{\lambda} \frac{\partial c_{D}}{\partial r_{i D}}=\omega \frac{\partial \psi_{D}}{\partial t_{D}},
\end{aligned}
$$

(flow in fracture).
Dimensionless initial and boundary conditions are given as follows.

The initial condition is

$$
\psi_{D}\left(r_{D}, t_{D}=0\right)=0 .
$$

The boundary condition is

$$
\frac{\partial \psi_{D}}{\partial r_{D}}\left(r_{D}=1, t_{D}\right)=-1 .
$$

The infinite outer boundary condition is

$$
\psi_{D}\left(r_{D} \longrightarrow \infty, t_{D}\right)=0 .
$$

The constant pressure boundary condition is

$$
\psi_{D}\left(r_{e D}, t_{D}\right)=0
$$

The closed boundary condition is

$$
\frac{\partial \psi_{D}}{\partial r_{D}}\left(r_{e D}, t_{D}\right)=0
$$

When $M=r_{i D} \mathcal{c}_{D}$, (13) can be transformed into

$$
\frac{\partial^{2} M}{\partial r_{i D}^{2}}=\lambda \frac{\partial M}{\partial t_{D}}
$$

The Laplace transform of (19) is

$$
\frac{\partial^{2} \bar{M}}{\partial r_{i D}^{2}}=\lambda u \bar{M} .
$$

The general solution of (20) is

$$
\bar{M}=A \sinh \left(\sqrt{\lambda u} r_{i D}\right)+B \cosh \left(\sqrt{\lambda u} r_{i D}\right),
$$

where $\sinh (x)=\left(e^{x}-e^{-x}\right) / 2$ and $\cosh (x)=\left(e^{x}+e^{-x}\right) / 2$.

The coefficients $A$ and $B$ could be obtained by the initial and boundary conditions:

$$
B=0, \quad A=\frac{\bar{M}_{a}}{\sinh (\sqrt{\lambda u})} .
$$

Hence,

$$
\bar{M}=\bar{M}_{a} \frac{\sinh \left(\sqrt{\lambda u} r_{i D}\right)}{\sinh (\sqrt{\lambda u})} .
$$

Therefore, the diffusion equation is transformed into

$$
\left.\frac{\partial \bar{c}_{D}}{\partial r_{i D}}\right|_{r_{i D}=1}=\bar{c}_{D}(\sqrt{\lambda u} \operatorname{coth} \sqrt{\lambda u}-1) \text {. }
$$

Combining the dimensionless definition $c_{D}$ and Langmuir isothermal adsorption equation $V=V_{L} P_{m} /\left(P_{L}+P_{m}\right)$, (24) is transformed into

$$
\left.\frac{\partial \bar{c}_{D}}{\partial r_{D}}\right|_{r_{D}=1}=L\left[\frac{V_{L} P}{P_{L}+P}-\frac{V_{L} P_{i}}{P_{L}+P_{i}}\right](\sqrt{\lambda u} \operatorname{coth} \sqrt{\lambda u}-1),
$$


where $L$ is Laplace transform:

$$
L\left[\frac{V_{L} P}{P_{L}+P}-\frac{V_{L} P_{i}}{P_{L}+P_{i}}\right]=\beta \bar{P}_{D}
$$

So,

$$
\left.\frac{\partial \bar{c}_{D}}{\partial r_{D}}\right|_{r_{D}=1}=-\beta \bar{P}_{D}(\sqrt{\lambda u} \operatorname{coth} \sqrt{\lambda u}-1) .
$$

Laplace transform of (20) is

$$
\frac{1}{r_{D}} \frac{\partial}{\partial r_{D}}\left(r_{D} \frac{\partial \bar{\psi}_{D}}{\partial r_{D}}\right)+\frac{(1-\omega)}{\lambda} \frac{\partial \bar{c}_{D}}{\partial r_{i D}}=\omega u \bar{\psi}_{D} .
$$

The initial conditions are

$$
\left.\bar{\psi}_{D}\right|_{u \rightarrow \infty}=0 .
$$

The inner boundary conditions are

$$
\left.r_{D} \frac{\partial \bar{\psi}_{D}}{\partial r_{D}}\right|_{r_{D}=1}=-\frac{1}{u}
$$

The outer boundary conditions are

$$
\begin{aligned}
& \left.\bar{\psi}_{D}\right|_{r_{D} \rightarrow \infty}=0 \quad \text { (infinite) }, \\
& \left.\bar{\psi}_{D}\right|_{r_{D}=r_{e D}}=0 \quad \text { (constant pressure), } \\
& \left.\frac{\partial \bar{\psi}_{D}}{\partial r_{D}}\right|_{r_{D}=r_{e D}}=0 \quad \text { (closed). }
\end{aligned}
$$

The solution of diffusion equation (27) is

$$
\left.\frac{\partial \bar{c}_{D}}{\partial r_{i D}}\right|_{r_{i D}=1}=\bar{c}_{D}(\sqrt{\lambda u} \operatorname{coth} \sqrt{\lambda u}-1) .
$$

Substituting the dimensionless definition $c_{D}$ and Langmuir isothermal adsorption equation $V=V_{L} P_{m} /\left(P_{L}+P_{m}\right)$ in (32), it can be written as follows:

$$
\left.\frac{\partial \bar{c}_{D}}{\partial r_{i D}}\right|_{r_{D}=1}=L\left[\frac{V_{L} P}{P_{L}+P}-\frac{V_{L} P_{i}}{P_{L}+P_{i}}\right](\sqrt{\lambda u} \operatorname{coth} \sqrt{\lambda u}-1) .
$$

Substituting $\psi_{D}=\left(\psi_{i}-\psi\right) / \psi_{i} q_{D}$ in (33), it can be expressed as follows:

$$
\frac{V_{L} P}{P_{L}+P}-\frac{V_{L} P_{i}}{P_{L}+P_{i}}=-\frac{\psi_{L} V_{L} \psi_{i} q_{D}}{\left(\psi_{L}+\psi\right)\left(\psi_{L}+\psi_{i}\right)\left(\psi+\psi_{i}\right)} \bar{\psi}_{D} .
$$

Defining $\beta=\psi_{L} V_{L} \psi_{i} q_{D} /\left(\psi_{L}+\psi\right)\left(\psi_{L}+\psi_{i}\right)\left(\psi+\psi_{i}\right)$,

$$
\left.\frac{\partial \bar{c}_{D}}{\partial r_{i D}}\right|_{r_{i D}=1}=-\beta \bar{\psi}_{D}(\sqrt{\lambda u} \operatorname{coth} \sqrt{\lambda u}-1) \text {. }
$$

Substituting (35) in (28),

$$
\begin{gathered}
\frac{1}{r_{D}} \frac{\partial}{\partial r_{D}}\left(r_{D} \frac{\partial \bar{\psi}_{D}}{\partial r_{D}}\right)=f(u) \bar{\psi}_{D}, \\
f(u)=\omega u+\frac{1-\omega}{\lambda} \beta(\sqrt{\lambda u} \operatorname{coth} \sqrt{\lambda u}-1) .
\end{gathered}
$$

\section{Equation of Boundary Condition}

4.1. Boundary Integral Equation. Applying the theory of boundary element, previous equation can be solved from the integral transformation of the governing equation based on the expression of the fundamental solutions and differential equation of fluid flow through porous medium:

$$
\begin{aligned}
& \int_{\Omega}\left[\psi_{D}(P, u) \nabla^{2} G(P, Q, u)-G(P, Q, u) \nabla^{2} \psi_{D}(P, u)\right. \\
& +\delta(P, Q) \psi_{D}(P, u)-\frac{1}{u} \sum_{i=1}^{N w} q_{D i} \delta\left(x_{D}-x_{D i}, y_{D}-y_{D i}\right) \\
& \cdot G(P, Q, u)] d \Omega=0
\end{aligned}
$$

where $G(P, Q, u)$ is the fundamental solution of horizontal wells in complex boundary reservoirs.

According to the properties of $\delta$ function and the second order of Green formula, it can be simplified to the boundary integral equation:

$$
\begin{aligned}
\psi_{D}\left(Q_{k}, u\right)=\int_{\Gamma}\left[G\left(P, Q_{k}, u\right) \frac{\partial \psi_{D}\left(P^{\prime}, u\right)}{\partial n}\right. \\
\left.\quad-\psi_{D}(P, u) \frac{\partial G\left(P^{\prime}, Q_{k}, u\right)}{\partial n}\right] d \Gamma\left(P^{\prime}\right) \\
+\frac{1}{u} \sum_{i=1}^{N w} q_{D i} G\left(P, Q_{i}, u\right) .
\end{aligned}
$$

The boundary $\Gamma$ is divided into $N_{b}$ cells, which are located at the end point and are taken as the nodes of boundary elements. Cell properties are assumed as linear distribution. Meanwhile, the boundary sections near nodes are assumed as arcs with nodes as their centers; the resulting boundary integral equation is

$$
\begin{aligned}
& \theta_{k} \psi_{D}\left(Q_{k}, u\right) \\
& =\sum_{i=1}^{N_{b}} \int_{\Gamma_{i}}\left[G\left(P^{\prime}, Q_{k}, u\right) \frac{\partial \psi_{D}\left(P^{\prime}, u\right)}{\partial n}\right. \\
& \left.-\psi_{D}\left(P^{\prime}, u\right) \frac{\partial G\left(P^{\prime}, Q_{k}, u\right)}{\partial n}\right] d \Gamma_{i}\left(P^{\prime}\right) \\
& +\frac{1}{u} \sum_{i=1}^{N w} q_{D i} G\left(P, Q_{i}, u\right),
\end{aligned}
$$


where $\theta_{k}$ represents interior angles between any two adjacent boundary elements. Consider

$$
\theta_{k}= \begin{cases}1, & \text { the point in domain } \theta_{i}=2 \pi, \\ 0.5, & \text { the point at smooth boundary } \theta_{i}=\pi, \\ \frac{\theta_{i}}{2 \pi}, & \text { the point at smoothless boundary. }\end{cases}
$$

Using the linear interpolation in boundary element, the boundary integral formula is deformed as follows:

$$
\begin{aligned}
& \theta_{k} \psi_{D}\left(Q_{k}, u\right) \\
& =\sum_{i=1}^{N_{b}} \frac{l_{i}}{2} \int_{-1}^{1}\left[G\left(P^{\prime}, Q_{k}, u\right)\left(\varphi_{1}(\xi) \frac{\partial \psi_{D i}}{\partial n}+\varphi_{2}(\xi) \frac{\partial \psi_{D i+1}}{\partial n}\right)\right. \\
& \left.\quad-\left(\varphi_{1}(\xi) \psi_{D i}+\varphi_{2}(\xi) \psi_{D i+1}\right) \frac{\partial G\left(P^{\prime}, Q_{k}, u\right)}{\partial n}\right] d \xi \\
& +\frac{1}{u} \sum_{i=1}^{N w} q_{D i} G\left(P^{\prime}, Q_{i}, u\right),
\end{aligned}
$$

where $\phi_{1}(\xi)=(1-\xi) / 2$ and $\phi_{2}(\xi)=(1+\xi) / 2$ are linear interpolation formula, $l_{i}=\sqrt{\left(x_{i+1}-x_{i}\right)^{2}+\left(y_{i+1}-y_{i}\right)^{2}},-1<$ $\xi<1$, and $\Gamma_{i}$ is the length of linearity cell.

4.2. Fundamental Solution of Boundary Element Integral Equation. It is crucial to find its fundamental solution when the horizontal flow problem in complex reservoirs is resolved using the boundary element method. According to the properties of the boundary element and the mathematic equation governing pressure transmission in porous medium, the fundamental solution must satisfy the modified Helmholtz operator. The equation is given by

$$
\frac{1}{r} \frac{\partial}{\partial r}\left(r \frac{\partial G}{\partial r}\right)-f(u) G=-2 \pi \delta\left(M_{D}, M_{D}^{\prime}\right) .
$$

With Lord Kelvin point source solution, the fundamental solution of (42) can be derived as follows:

$$
\bar{\gamma}=\frac{\exp \left(-\rho_{D} \sqrt{f(u)}\right)}{4 \pi \rho_{D}} .
$$

With mirror image method of fluid mechanics in porous medium, the transient point source fundamental solution of closed boundary is

$$
\begin{gathered}
\bar{\gamma}=\frac{1}{4 \pi} \sum_{-\infty}^{+\infty}\left\{\frac{\exp \left(-\sqrt{f(u)} \sqrt{R_{D}^{2}+\left(Z_{D}+Z_{D}^{\prime}-2 n Z_{e D}\right)^{2}}\right)}{\sqrt{R_{D}^{2}+\left(Z_{D}+Z_{D}^{\prime}-2 n Z_{e D}\right)^{2}}}\right. \\
\left.+\frac{\exp \left(-\sqrt{f(u)} \sqrt{R_{D}^{2}+\left(Z_{D}-Z_{D}^{\prime}-2 n Z_{e D}\right)^{2}}\right)}{\sqrt{R_{D}^{2}+\left(Z_{D}-Z_{D}^{\prime}-2 n Z_{e D}\right)^{2}}}\right\} .
\end{gathered}
$$

With Poisson superposition formula, (44) can be simplified, and the transient point source fundamental solution of sealed boundary at $Z=0$ and $Z=Z e$ is

$$
\begin{aligned}
\bar{\gamma}=\frac{1}{2 \pi Z_{e D}}[ & K_{0}\left(R_{D} \sqrt{f(u)}\right) \\
& +2 \sum_{n=1}^{n=\infty} K_{0}\left(R_{D} \sqrt{f(u)+\frac{n^{2} \pi^{2}}{Z_{e D}^{2}}}\right) \\
& \left.\cdot \cos \left(n \pi \frac{Z_{D}}{Z_{e D}}\right) \cos \left(n \pi \frac{Z_{D}^{\prime}}{Z_{e D}}\right)\right] .
\end{aligned}
$$

The fundamental boundary element solution of horizontal wells in a reservoir with closed top and bottom boundaries is

$$
\begin{aligned}
& G\left(P^{\prime}, Q, u\right) \\
& =\frac{1}{2} \int_{-1}^{1} K_{0}\left(R_{D} \sqrt{f(u)}\right) d \alpha \\
& +\sum_{n=1}^{n=\infty} \cos \left(n \pi z_{D}\right) \cos \left(n \pi z_{w D}\right) \\
& \cdot \int_{-1}^{1} K_{0}\left(\sqrt{\left(x_{D}-\alpha\right)^{2}+y_{D}^{2}} \sqrt{f(u)+\frac{n^{2} \pi^{2}}{Z_{e D}^{2}}}\right) d \alpha, \\
& \frac{\partial G\left(P^{\prime}, Q, u\right)}{\partial n} \\
& =-\frac{1}{2} \int_{-1}^{1} \sqrt{f(u)} K_{1}\left(\left(r_{D}-\alpha\right) \sqrt{f(u)}\right) \frac{\partial r_{D}}{\partial n} d \alpha \\
& -\sum_{n=1}^{n=\infty} \cos \left(n \pi z_{D}\right) \\
& \cdot \cos \left(n \pi z_{w D}\right) \int_{-1}^{1} \sqrt{f(u)+\frac{n^{2} \pi^{2}}{Z_{e D}^{2}}} \\
& K_{1}\left(\left(r_{D}-\alpha\right)\right. \\
& \left.\cdot \sqrt{f(u)+\frac{n^{2} \pi^{2}}{Z_{e D}^{2}}}\right) \frac{\partial r_{D}}{\partial n} d \alpha
\end{aligned}
$$

where

$$
\begin{gathered}
\frac{\partial r_{D}}{\partial n}= \pm \mid\left(\left(x_{\xi}-x\right)\left(y_{i}-y_{i+1}\right)-\left(y_{\xi}-y\right)\left(x_{i}-x_{i+1}\right)\right) \\
\cdot\left(\sqrt{\left(x_{i}-x_{i+1}\right)^{2}+\left(y_{i}-y_{i+1}\right)^{2}}\right)^{-1} \mid \\
\cdot\left(\sqrt{\left(x_{\xi}-x_{i}\right)^{2}+\left(y_{\xi}-y_{i}\right)^{2}}\right)^{-1} .
\end{gathered}
$$


If $n$ and $P^{\prime}$ are in the same direction, then $\partial r_{D} / \partial n$ is positive; otherwise it is negative.

4.3. Solving of Integral Equation. The integral boundary equation (37) can be simplified as

$$
\begin{aligned}
\theta_{k} \psi_{D}\left(Q_{k}, u\right) & \\
=\sum_{i=1}^{N_{b}}\left(H_{k 1}^{\prime} \frac{\partial \psi_{D i}}{\partial n}+H_{k 2}^{\prime} \frac{\partial \psi_{D i+1}}{\partial n}\right. & \\
& \left.\quad+H_{k 3}^{\prime} \psi_{D i}+H_{k 4}^{\prime} \psi_{D i+1}\right)+\frac{1}{u} \sum_{i=1}^{N w} q_{D i} G\left(P^{\prime}, Q, u\right),
\end{aligned}
$$

where

$$
\begin{aligned}
& H_{1}^{\prime}=\frac{l_{i}}{2} \int_{-1}^{1} G\left(P^{\prime}, Q_{k}, u\right) \phi_{1}(\xi) d \xi ; \\
& H_{2}^{\prime}=\frac{l_{i}}{2} \int_{-1}^{1} G\left(P^{\prime}, Q_{k}, u\right) \phi_{2}(\xi) d \xi ; \\
& H_{3}^{\prime}=\frac{l_{i}}{2} \int_{-1}^{1}-\frac{\partial G\left(P^{\prime}, Q_{k}, u\right)}{\partial n} \phi_{1}(\xi) d \xi ; \\
& H_{4}^{\prime}=\frac{l_{i}}{2} \int_{-1}^{1}-\frac{\partial G\left(P^{\prime}, Q_{k}, u\right)}{\partial n} \phi_{2}(\xi) d \xi
\end{aligned}
$$

The unknown variable in the integral boundary equation is $\partial \psi_{D i} / \partial n$ or $\psi_{D i}$. The number of the nodes at the boundary is $N_{b}$, so $N_{b}$ equation with form of (48) can be established. When the boundary properties are known, there are just $N_{b}$ unknown variables. So we can solve the set of equations, whose matrix expression is

$$
\left[\begin{array}{cccc}
H_{11} & H_{12} & \cdots & H_{1 N_{b}} \\
H_{21} & H_{22} & \cdots & H_{2 N_{b}} \\
\vdots & \vdots & \vdots & \vdots \\
H_{N b 1} & H_{N b 2} & \cdots & H_{N_{b} N_{b}}
\end{array}\right]\left[\begin{array}{c}
x_{1} \\
x_{2} \\
\vdots \\
x_{N b}
\end{array}\right]=\left[\begin{array}{c}
F_{1} \\
F_{2} \\
\vdots \\
F_{N b}
\end{array}\right],
$$

where $x_{i}$ is $\partial \psi_{D i} / \partial n$ or $\psi_{D i}$ and $F_{i}$ is $(1 / u) \sum_{i=1}^{N w} q_{D i} G\left(P^{\prime}, Q, u\right)$.

Once the unknown variables are acquired, we can solve $P_{D}$ of arbitrary point in the research domain using the boundary integral equation (50):

$$
\begin{aligned}
\psi_{D}(Q, u)=\sum_{i=1}^{N_{b}}\left(H_{k 1}^{\prime} \frac{\partial \psi_{D i}}{\partial n}+H_{k 2}^{\prime} \frac{\partial \psi_{D i+1}}{\partial n}+H_{k 3}^{\prime} \psi_{D i}\right. \\
\left.+H_{k 4}^{\prime} \psi_{D i+1}\right)+\frac{1}{u} \sum_{i=1}^{N w} q_{D i} G\left(P^{\prime}, Q, u\right) .
\end{aligned}
$$

\section{Validation of the Model}

In order to validate the gas flow model proposed in this paper, we test the adsorption and desorption data of coal. Then, we compare the boundary element model with those published works.

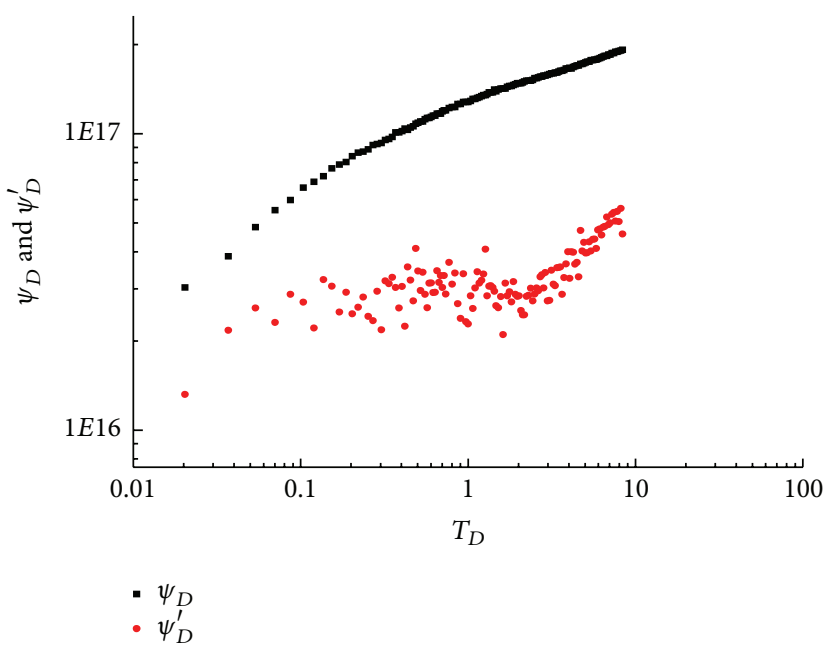

Figure 4: Double logarithmic curve of pressure drop of test data.

5.1. Validation of the Gas Flow Model in CBM Reservoirs. The adsorption and desorption data of coal are tested in the laboratory; the pressure continues to drop as the coal continues to adsorb methane. We deal with the experimental data in $\log -\log$ coordinates as shown in Figure 4. It can be seen from the diagram that the results of the numerical simulation are in good match with the experimental data and are consistent with the gas flow law in CBM reservoirs.

5.2. Validation of Gas Flow in a Horizontal Well with Complex Boundary. A pressure buildup test example of a horizontal gas well in tight gas reservoir has been presented by Han [30]; the production and pressure data fitting results show that the horizontal gas well has a closed boundary.

Figure 5 is the comparison of pressure curves between horizontal wells in CBM and conventional gas reservoirs, and the values of parameters are from Han's paper [30]. According to the analysis of flow characteristics, there is an additional radial flow which reflects gas flow in fracture. After this stage, the pressure transmits from fracture to matrix and gas starts to desorb. The flow characteristics of this stage would be influenced by desorption velocity and desorption quantity. The last stage of pressure derivative curves in different reservoirs is in good match, which reflects the type of boundary.

\section{Analysis of Flow Characteristics and Field Application}

6.1. Flow Regime Recognition. Type curves for horizontal wells in a CBM reservoir with complex boundary calculated by the BEM in this paper are shown in Figure 6. The figure shows that flow characteristics can be divided into seven flow periods: (1) wellbore storage period; influenced by early wellbore storage effect, the curves of pressure and pressure derivative are straight lines with the same slope in this period; (2) the first transition flow section, which reflects the degree of pollution near the bottom and is mainly affected by skin 


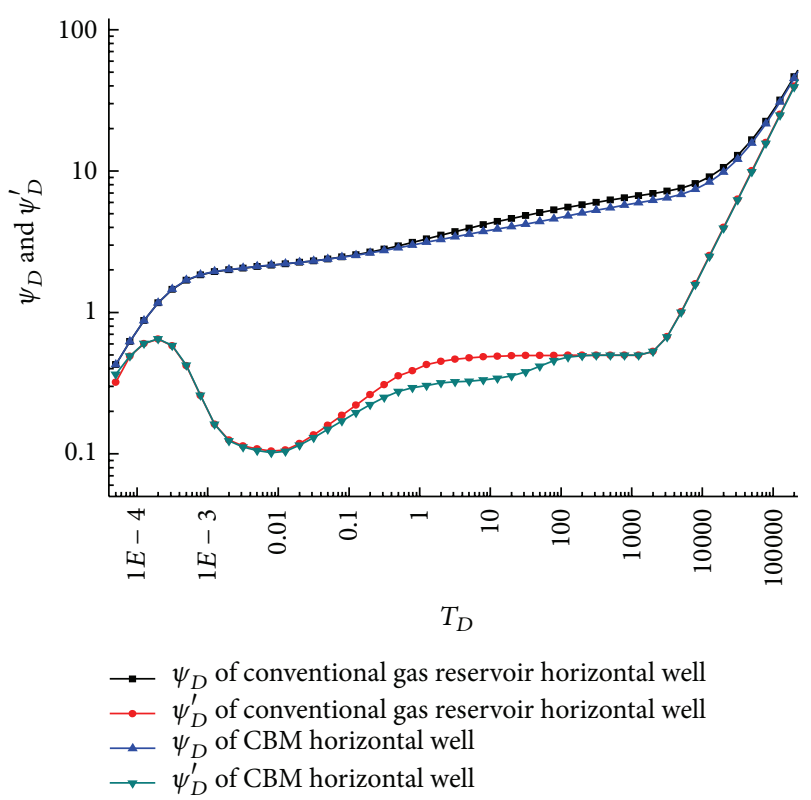

FIGURE 5: Comparison of typical curves between horizontal wells in $\mathrm{CBM}$ and conventional gas reservoirs.

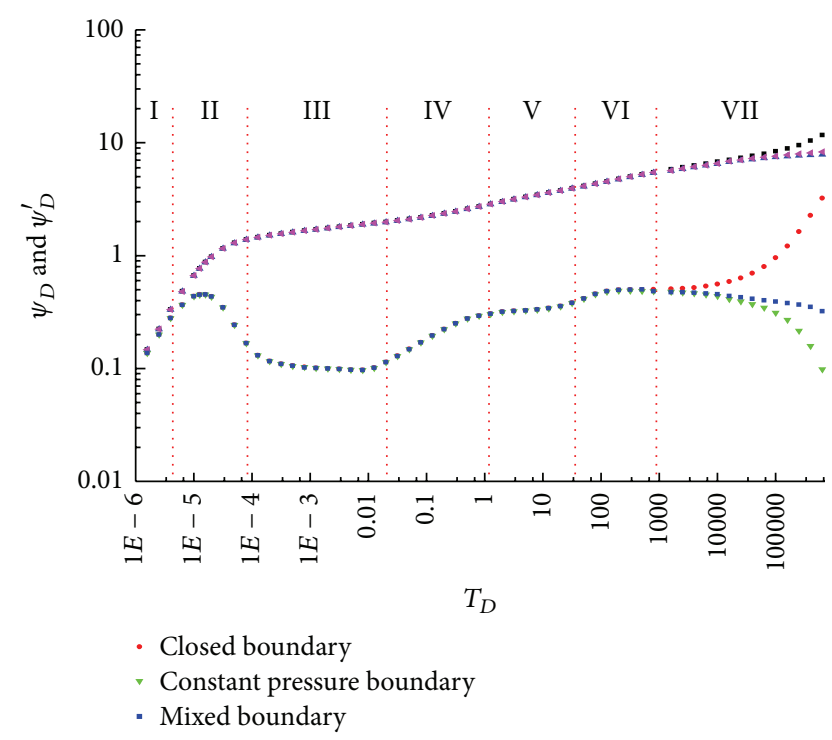

Figure 6: Typical curve of CBM horizontal well with complex boundary conditions $\left(\omega=0.01, \lambda=300, \beta=50, L_{D}=2.5\right.$, $Z_{w d}=0.5, C_{d}=0.0001, s=1$, and $\left.r_{e D}=50\right)$.

factor $S$; (3) the first radial flow section, which is perpendicular to the horizontal wellbore; the pressure derivative curve is shown as a horizontal line, which reflects the early radial flow perpendicular to the horizontal wellbore; (4) the second transition section, which is the transition stage of radial flow from wellbore to fracture; (5) the second radial flow stage, which reflects the free gas flow in fracture; this period is affected by the flow of free gas and adsorbed gas, diffusion coefficient $\lambda$, and Langmuir adsorption parameter $\beta$; (6) the third radial flow section, which reflects radial flow of whole

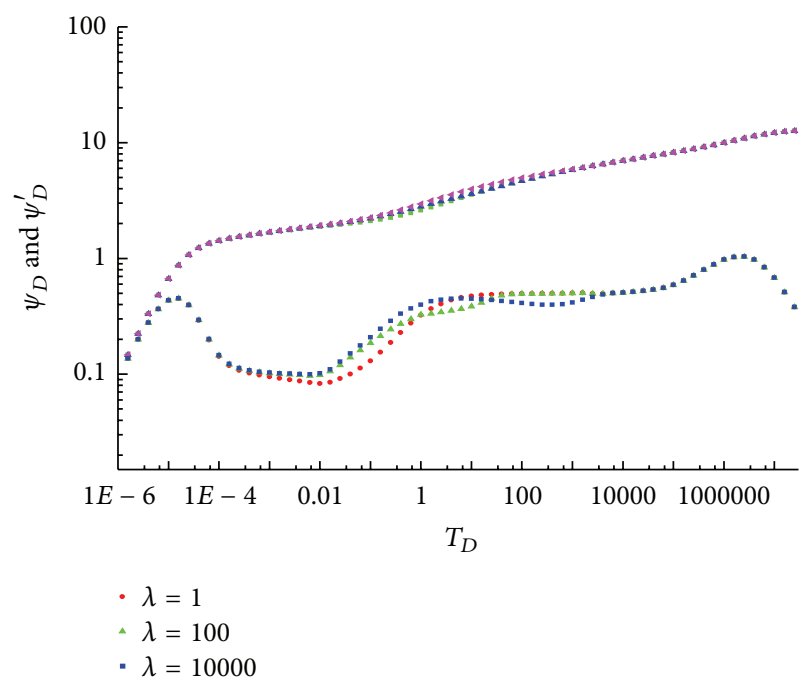

Figure 7: Influence of diffusion coefficient $\lambda$ on bottom-hole pressure with mixed boundary $\left(\omega=0.01, \beta=50, L_{D}=2.5\right.$, $Z_{w d}=0.5, C_{d}=0.0001, s=1$, and $\left.r_{e D}=50\right)$.

system after pressure balance, and pressure derivative curve appears as a flat behavior; (7) boundary response section. The pressure and pressure derivative curves with closed boundary would be upward after pressure transmitting to the boundary. The pressure derivative curve with constant pressure boundary would be in decline after pressure transmitting to the boundary. Compared with the constant pressure boundary, the falling range of pressure derivative curve with mixed boundary is less.

6.2. Parameter Sensitivity Analysis. Figure 7 shows the influence of diffusion coefficient $\lambda$ on the bottom-hole pressure with mixed boundary. There is relevance between diffusion coefficient $\lambda=\alpha k \tau / \theta L^{2}$ and adsorption time $\tau=R^{2} / D$. The smaller the $\tau$, the shorter the time of desorption-diffusion and the shorter the time of pressure balance between fracture and matrix. As shown in the diagram, diffusion coefficient $\lambda$ mainly affects the duration of the second radial flow period and appearance of third radial flow. The greater the diffusion coefficient $\lambda$, the longer the duration of second radial flow period and the later the $t$ appearance of third radial flow, and vice versa. If $\lambda$ is small enough, the second radial flow period would be covered.

Figure 8 shows the influence of Langmuir parameter $\beta$ on the bottom-hole pressure with mixed boundary. $\beta=$ $\psi_{L} V_{L} \psi_{i} q_{D} /\left(\psi_{L}+\psi\right)\left(\psi_{L}+\psi_{i}\right)\left(\psi+\psi_{i}\right)$ is related to Langmuir adsorption pressure $p_{L}$ and Langmuir adsorption volume $V_{L}$. The larger the $\beta$, the greater the adsorption ability of coal. As shown in the graph, $\beta$ only affects the second radial flow section. The greater the $\beta$, the smaller the pressure derivative values of second radial flow period.

Figure 9 shows the influence of fracture storage ratio $\omega$ on the bottom-hole pressure with mixed boundary. Its expression $\omega=\phi_{f} c_{t} \mu /\left(\phi_{f} c_{t} \mu+6 p_{s c} T / q_{D} T_{s c} \psi_{i}\right)$ indicates that the smaller the $\phi_{f} \mathcal{c}_{t}$, the smaller the $\omega$ and the more the radial 


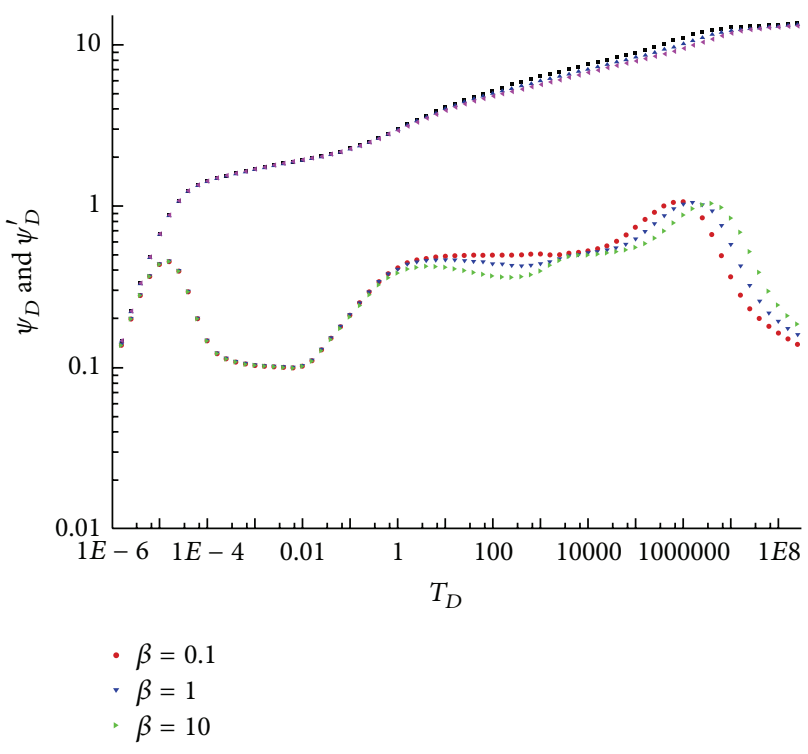

FIGURE 8: Influence of $\beta$ on the bottom-hole pressure with mixed boundary $\left(\omega=0.01, \lambda=300, L_{D}=2.5, Z_{w d}=0.5, C_{d}=0.0001\right.$, $s=1$, and $\left.r_{e D}=50\right)$.

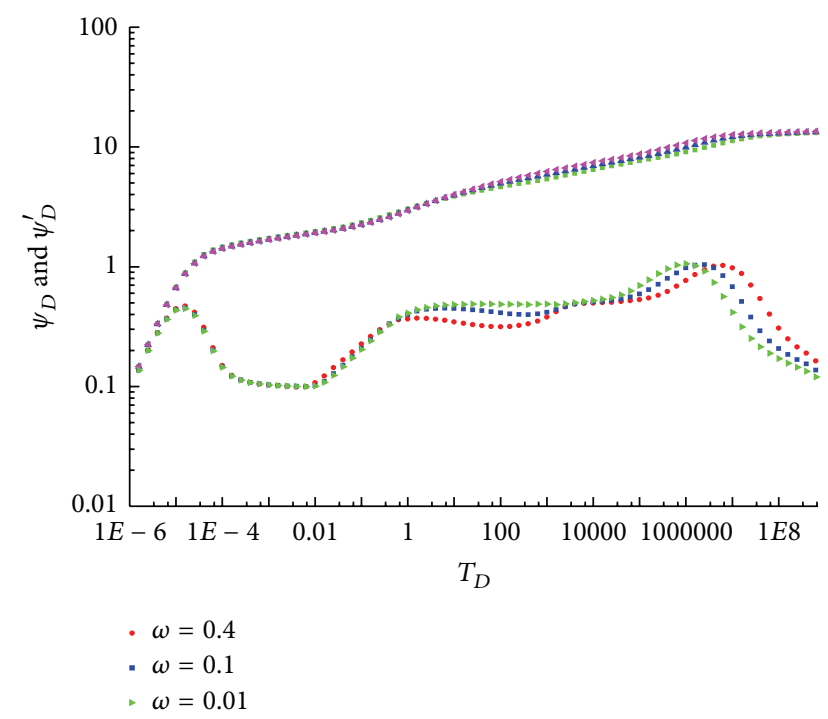

FIGURE 9: Influence of $\omega$ on the bottom-hole pressure with mixed boundary $\left(\lambda=300, \beta=50, L_{D}=2.5, Z_{w d}=0.5, C_{d}=0.0001\right.$, $s=1$, and $\left.r_{e D}=50\right)$.

flow in fracture. From Figure 8, almost all of the flow periods would be affected by $\omega$, except for wellbore storage period. The greater the $\omega$, the smaller the hump value of pressure derivative, and the longer the duration of first radial flow period, the greater the pressure derivative value in second radial flow period.

Figure 10 shows the influence of eccentricity of horizontal well $Z_{w d}$ on the bottom-hole pressure with mixed boundary. The streamline shape and pressure distribution of a horizontal well would be affected by the size of $Z_{w d}$. The smaller

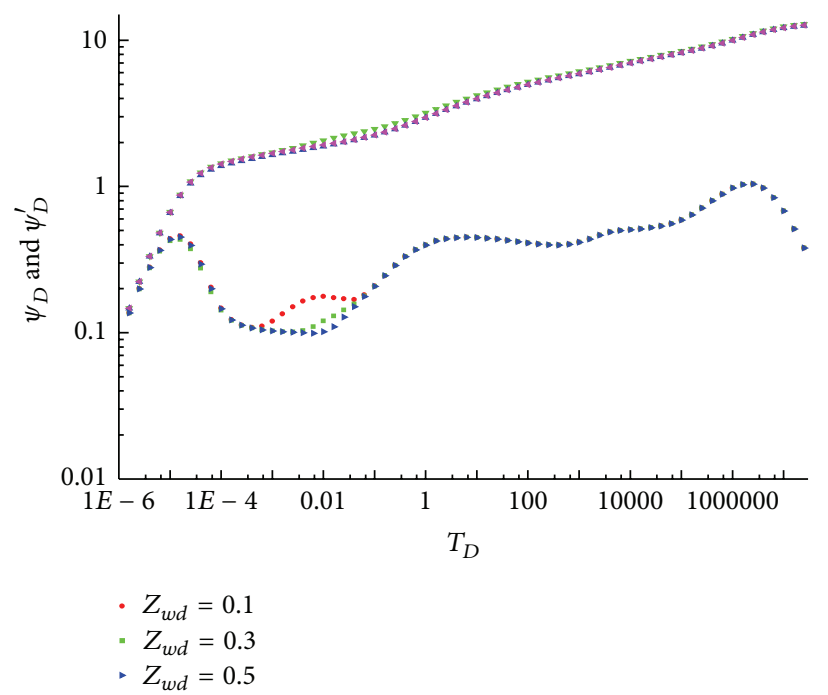

FIGURE 10: Influence of $Z_{w d}$ on the bottom-hole pressure with mixed boundary $\left(\omega=0.01, \lambda=300, \beta=50, L_{D}=2.5, C_{d}=0.0001, s=1\right.$, and $\left.r_{e D}=50\right)$.

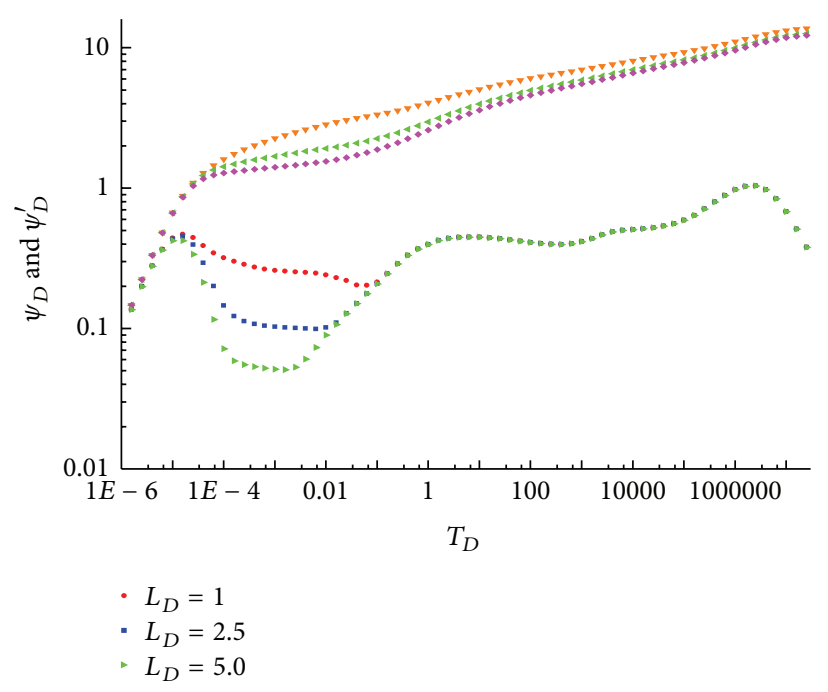

FIGURE 11: Influence of $L_{D}$ on the bottom-hole pressure with mixed boundary $\left(\omega=0.01, \lambda=300, \beta=50, Z_{w d}=0.5, C_{d}=0.0001, s=1\right.$, and $\left.r_{e D}=50\right)$.

the $Z_{w d}$, the larger the pressure derivative value in first radial flow period.

Figure 11 shows the influence of length of horizontal well $L_{D}$ on the bottom-hole pressure with mixed boundary. It shows large influence of $L_{D}$ on the pressure derivative value in first radial flow period. The smaller the $L_{D}$, the larger the pressure derivative value in first radial flow period.

6.3. Field Example. To demonstrate the application of the model proposed in this paper, a horizontal well ZX-12 in a CBM reservoir is studied. The vertical depth and horizontal length of this well are $689 \mathrm{~m}$ and $536 \mathrm{~m}$, respectively, with well radius $r_{w}$ of $0.1 \mathrm{~m}$, coal thickness of $4.5 \mathrm{~m}$, and initial 


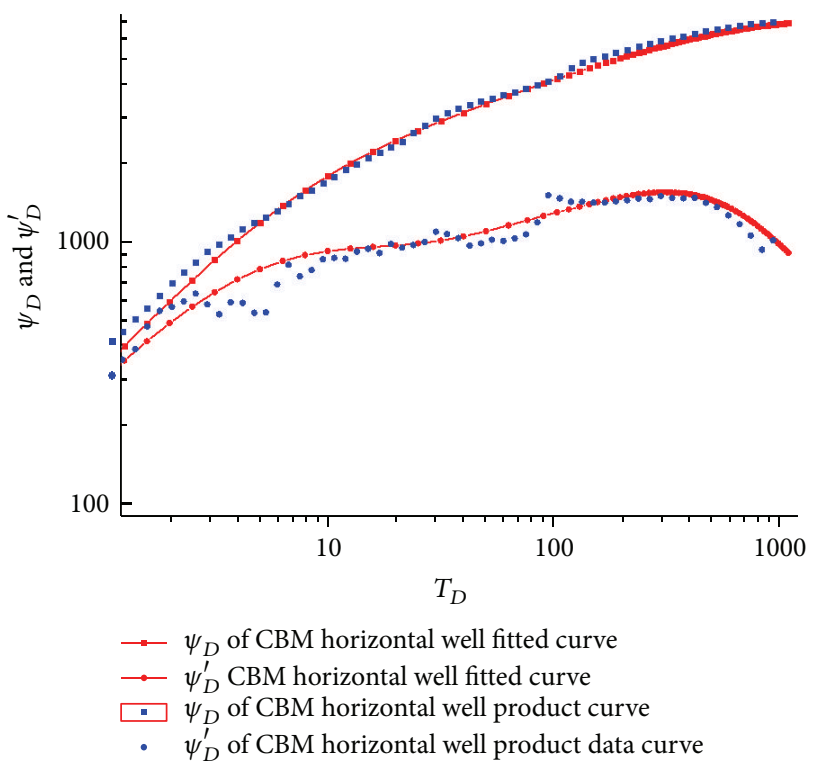

FIGURE 12: Fitted curves of test data from an actual well.

pressure of 5.5 $\mathrm{MPa}$. According to test results, the Langmuir volume $V_{L}$ is $32.75 \mathrm{~m}^{3} / \mathrm{t}$ and the Langmuir pressure $P_{L}$ is $2.49 \mathrm{MPa}$. Figure 12 is the fitted curves which were obtained by calculating the actual production and pressure data. As shown in Figure 12, the field data are in good match with the theoretical results calculated by the new model in this paper.

According to the actual field data, some of reservoir parameters are fitted as follows: the porosity is 0.04 and the permeability is $3 \mathrm{mD}$. The stage of desorption and boundary response can be clearly seen in Figure 12. Because of the falling of the pressure derivative curve in the last stage, well ZX-12 has a constant pressure boundary, implying that the gas production of well ZX-12 is affected by the production of adjacent well.

\section{Conclusions}

The mathematic model of gas flowing into horizontal wells in CBM reservoirs with complex boundary conditions is derived based on the percolation theory. The curves of bottomhole pressure and pressure derivative are obtained by using boundary element method and Laplace transform. The conclusions are as follows.

(1) The fundamental boundary element solutions for transient pressure response of horizontal wells in CBM reservoirs with complex boundary conditions could be obtained by using Lord Kelvin's point source solution, point source function theory, and Poisson's summation formula.

(2) Comparison of the typical curves of flow characteristics between horizontal wells in CBM and conventional gas reservoirs shows that there is an additional radial flow which reflects gas flow in fracture.

(3) Comparing the typical curves of flow characteristics with complex boundary conditions, the pressure and pressure derivative curves with closed boundary would be upward after pressure transmitting to the boundary. The pressure derivative curves with constant pressure boundary and mixed boundary would be fallen, but the falling range of pressure derivative curve with mixed boundary is less.

\section{Nomenclature}

$r_{w}:$ Radius, $\mathrm{m}$

$k$ : Permeability, $\mathrm{mD}$

$\phi$ : Porosity, fraction

$J$ : Diffusion flux, $\mathrm{g} / \mathrm{m}^{2} \mathrm{~s}$

$s$ : Laplace transform variable

$h$ : Thickness, $\mathrm{m}$

$B$ : Volume factor, fraction

$q$ : Influx into the wellbore, $\mathrm{m}^{3} / \mathrm{d}$

$L$ : Half length of horizontal well, $\mathrm{m}$

$\mu$ : Viscosity, $\mathrm{mPa} \cdot \mathrm{s}$

$c$ : Concentration of coalbed methane, $\mathrm{kg} / \mathrm{m}^{3}$

$P_{i}$ : Initial pressure, $\mathrm{MPa}$

$q$ : Production of well, $\mathrm{m}^{3} / \mathrm{d}$

$V_{L}$ : Langmuir volume constant, $\mathrm{m}^{3} /$ ton

$V$ : Volume of coal matrix, $\mathrm{m}^{3}$

$\psi_{i}$ : Pseudopressure

$\omega$ : Fracture storage ratio, fraction.

\section{Conflict of Interests}

The authors declare that there is no conflict of interests regarding the publication of this paper.

\section{Acknowledgments}

This study is supported by National Natural Science Foundation of China (Grant no. 51304032), National Major Science and Technology Special Project of China (Grant no. 2011ZX05037-003), and Research Fund for the Doctoral Program of Higher Education of China (Grant no. 20125122110017).

\section{References}

[1] J. C. Cai, E. Perfect, C.-L. Cheng, and X. Y. Hu, "Generalized modeling of spontaneous imbibition based on hagen-poiseuille flow in tortuous capillaries with variably shaped apertures," Langmuir, vol. 30, no. 18, pp. 5142-5151, 2014.

[2] T. Ertekin and W. Sung, "Pressure transient analysis of coal seams in the presence of multi-mechanistic flow and sorption phenomena," in Proceedings of the SPE Gas Technology Symposium, pp. 469-477, June 1989.

[3] K. Anbarci and T. Ertekin, "A comprehensive study of pressure transient analysis with sorption phenomena for single phase gas flow in coal seams," SPE 20568, 1990.

[4] C. R. Clarkson and R. M. Bustin, "Effect of pore structure and gas pressure upon the transport properties of coal: a laboratory and modeling study. 1 . Isotherms and pore volume distributions," Fuel, vol. 78, no. 11, pp. 1333-1344, 1999. 
[5] C. R. Clarkson and R. M. Bustin, "Effect of pore structure and gas pressure upon the transport properties of coal: a laboratory and modeling study. 2. Adsorption rate modeling," Fuel, vol. 78, no. 11, pp. 1345-1362, 1999.

[6] S. Reeve, "Advanced reservoir modeling in desorption controlled reservoirs," in Proceedings of the SPE Rocky Mountain Petroleum Technology Conference, SPE 71090, May 2001.

[7] D. K. Tong and S. Liu, "Unsteady percolation flow of coalbed methane through deformed coal seam," Natural Gas Industry, vol. 1, no. 1, pp. 74-76, 2005.

[8] X. M. Zhang and D. K. Tong, "Analysis for pressure transient of coalbed methane reservoir," Well Testing, vol. 6, no. 17, pp. 1-4, 2008.

[9] X. M. Zhang and D. K. Tong, "Pressure transient analysis for coal seams with triple-porosity/dual-permeability model," Chinese Quarterly of Mechanics, vol. 4, no. 29, pp. 578-582, 2008.

[10] X. H. Hu, S. M. Hu, D. I. Zhang et al., "Application of quadratic pressure method in well test interpretation of gas water two phase flow in coal seam," Journal of Oil and Gas Technology, vol. 7, no. 33, pp. 118-122, 2011.

[11] C. R. Clarkson, C. L. Jordan, D. Ilk, and T. A. Blasingame, "Ratetransient analysis of 2-phase (gas + water) CBM wells," Journal of Natural Gas Science and Engineering, vol. 8, pp. 106-120, 2012.

[12] K. Aminian and S. Ameri, "Predicting production performance of CBM reservoirs," Journal of Natural Gas Science and Engineering, vol. 1, no. 1-2, pp. 25-30, 2009.

[13] J. C. Cai and B. M. Yu, "A discussion of the effect of tortuosity on the capillary imbibition in porous media," Transport in Porous Media, vol. 89, no. 2, pp. 251-263, 2011.

[14] W. Sung, T. Ertekin, and F. C. Schwerer, "The development, testing, and application of a comprehensive coal seam degasification model," in Proceedings of the SPE Unconventional Gas Technology Symposium, SPE 15247, Louisville, Ky, USA, May 1986.

[15] W. Sung and T. Ertekin, "An analysis of field development strategies for methane production from coal seams," in Proceedings of the 62nd Annual Technical Conference and Exhibition, Conference Paper SPE 16858, Dallas, Tex, USA, 1987.

[16] T. Ertekin, W. Sung, and F. C. Schwerer, "Production performance analysis of horizontal drainage wells for degasification of coal-seams," Journal of Petroleum Technology, vol. 40, no. 5, pp. 625-632, 1988.

[17] T. W. Engler and J. M. Rajtar, "Pressure transient testing of horizontal wells in coalbed reservoirs," in Proceedings of the SPE Rocky Mountain Regional Meeting, Paper SPE-24374-MS, Casper, Wyo, USA, May 1992.

[18] P. S. Sarkar and J. M. Rajtar, "Horizontal well transient pressure testing in coalbed reservoirs," in Proceedings of the $3 \mathrm{rd}$ Latin America/Caribbean Petroleum Engineering Conference, SPE 26995, Buenos Aires, Argentina, April 1994.

[19] P. S. Sarkar and J. M. Rajtar, "Transient well testing of coalbed methane reservoirs with horizontal wells," in Proceedings of the Permian Basin Oil \& Gas Recovery Conference, Paper SPE27681, pp. 531-539, Midland, Tex, USA, March 1994.

[20] X. H. Wang, D. I. Zhang, and Y. Song, "Development mechanism of low permeable coalbed methane from multilateral horizontal pinnate well with non-darcy seepage flow characteristic," Acta Geologica Sinica, vol. 7, no. 82, pp. 1437-1443, 2008.

[21] R.-S. Nie, Y.-F. Meng, J.-C. Guo, and Y.-L. Jia, "Modeling transient flow behavior of a horizontal well in a coal seam," International Journal of Coal Geology, vol. 92, pp. 54-68, 2012.
[22] K. L. Katsifarakis, D. K. Karpouzos, and N. Theodossiou, "Combined use of BEM and genetic algorithms in groundwater flow and mass transport problems," Engineering Analysis with Boundary Elements, vol. 23, no. 7, pp. 555-565, 1999.

[23] D. T. Numbere and D. Tiab, "Improved streamline-generating technique that uses the boundary (integral) element method," SPE Reservoir Engineering, vol. 3, no. 3, pp. 1061-1068, 1988.

[24] J. Kikani and R. N. Horne, "Application of boundary element method to reservoir engineering problems," Journal of Petroleum Science and Engineering, vol. 3, no. 3, pp. 229-241, 1989.

[25] J. Hou, Y. D. Wang, and Y. M. Chen, "Boundary element method in enhanced oil recovery," Chinese Journal of Hydrodynamics, vol. 3, no. 13, pp. 23-28, 2003.

[26] K. Chaiyo, P. Rattanadecho, and S. Chantasiriwan, "The method of fundamental solutions for solving free boundary saturated seepage problem," International Communications in Heat and Mass Transfer, vol. 38, no. 2, pp. 249-254, 2011.

[27] K. Rafiezadeh and B. Ataie-Ashtiani, "Seepage analysis in multi-domain general anisotropic media by three-dimensional boundary elements," Engineering Analysis with Boundary Elements, vol. 37, no. 3, pp. 527-541, 2013.

[28] X. H. Fu, Y. Qin, W. H. Zhang et al., "Coal pore fractal classification and natural classification research based on the coal-bed gas migration," Chinese Science Bulletin, vol. 12, supplement 1, pp. 51-55, 2005.

[29] J. C. Cai and S. Y. Sun, "Fractal analysis of fracture increasing spontaneous imbibition in porous media with gas-saturated," International Journal of Modern Physics C, vol. 24, no. 8, pp. 135156, 2013.

[30] D. M. Han, The Dynamic Analysis Technology Research on Horizontal Wells of Jingbian Gas Field, University of Xian Shiyou, Xian, China, 2011. 

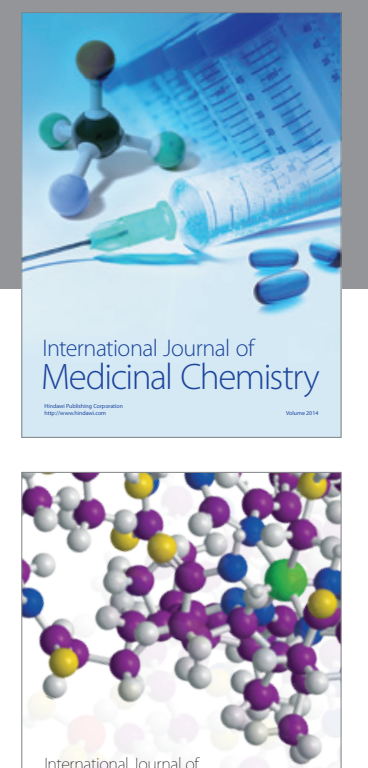

\section{Carbohydrate} Chemistry

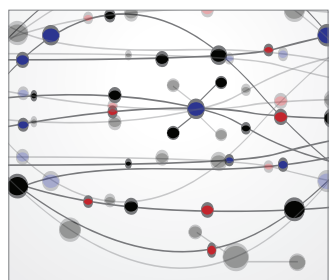

The Scientific World Journal
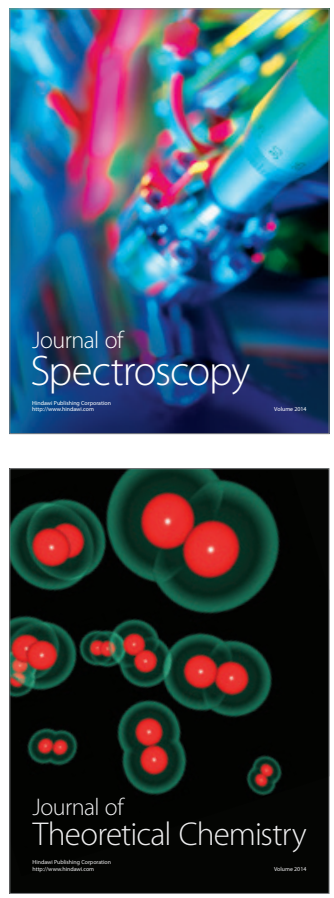
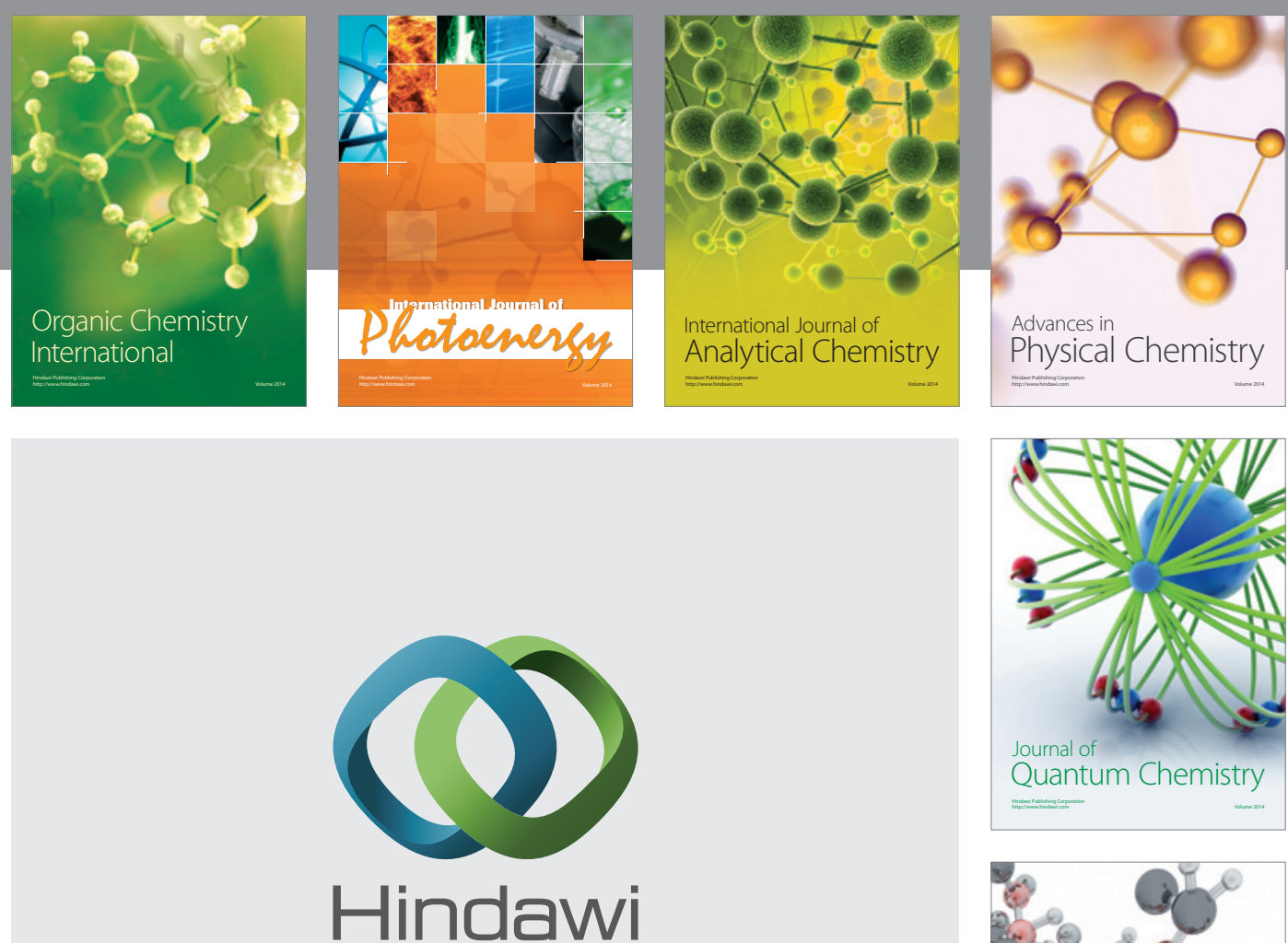

Submit your manuscripts at

http://www.hindawi.com

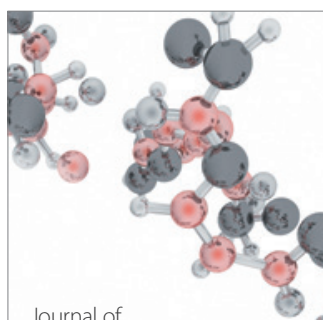

Analytical Methods

in Chemistry

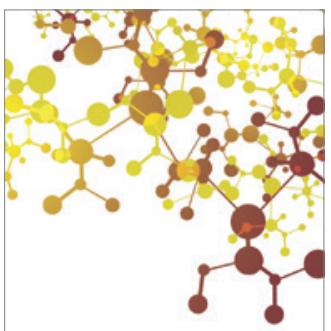

Journal of

Applied Chemistry

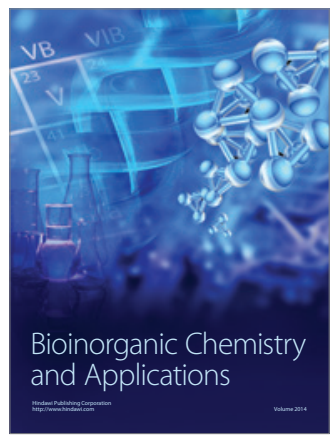

Inorganic Chemistry
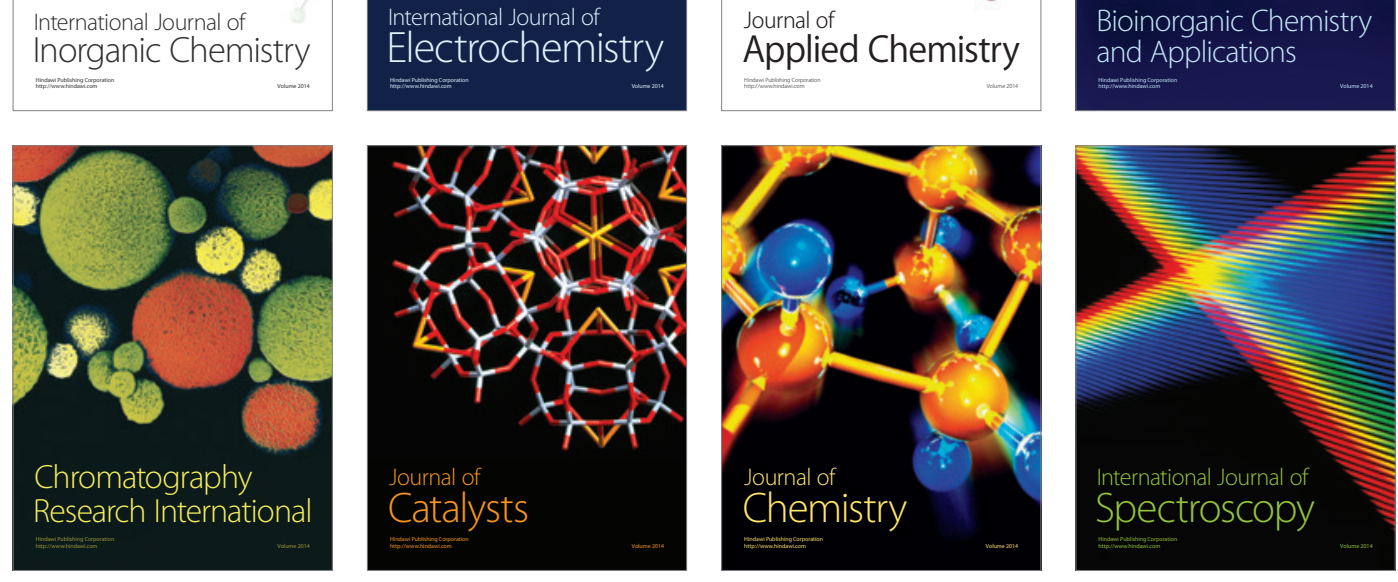